\title{
Tuberculosis diagnosis after bleach processing for early stage tuberculosis laboratory capacity building
}

\author{
S. David,* A. F. Sutre, ${ }^{*}$ A. Sanca, ${ }^{\dagger}$ A. Mané, ${ }^{\dagger}$ V. Henriques, ${ }^{\dagger}$ C. Portugal, ${ }^{*}$ L. Sancho, ${ }^{\ddagger}$ A. Cardoso, ${ }^{*}$ \\ E. Paixão,* E. L. Duarte,§ C. Q. F. Leite," J. I. Salem,\# A. Antunes** \\ * Instituto Nacional de Saúde Dr. Ricardo Jorge (INSA,IP), Lisbon, Portugal; ' Cumura Hospital, Cumura, Republic of \\ Guinea-Bissau; ¥Serviço de Patologia Clínica, Hospital Fernando Fonseca, Amadora, Portugal; § Escola de Ciências e \\ Tecnologia, Instituto de Ciências Agrárias e Ambientais Mediterrânicas, Universidade de Évora, Évora, Portugal; \\ "Faculty of Pharmaceutical Science, Universidade Estadual Paulista, Araraquara, São Paulo, Brazil; "Instituto Nacional \\ de Pesquisas da Amazônia, Manaus, Brazil; ** Instituto de Higiene e Medicina Tropical (IHMT), Universidade Nova de \\ Lisboa, Lisbon, Portugal
}

S U M M A R Y

The diagnosis of tuberculosis is seriously hampered in the absence of standard biosafety laboratory facilities for specimen concentration and Mycobacterium tuberculosis culture. Within a laboratory twinning arrangement, heat-fixed direct smear and sediment from 74 bleach-processed and 20 non-processed specimens from Cumura Hospital, Guinea-Bissau, were sent to Lisbon for molecular evaluation of rifampicin resistance. Sequence analysis of a 369 base-pair $r p o B$ locus detected
$3.2 \%(3 / 94)$ resistant specimens. To our knowledge, this represents the first report on the molecular analysis of M. tuberculosis from bleach-processed sputum, an alternative to current diagnostic practice in low-resource settings.

KEY WORDS: Mycobacterium tuberculosis; rpoB; sputum smear microscopy; sodium hypochlorite; denaturing high-performance liquid chromatography (DHPLC) sequencing
TUBERCULOSIS (TB) is a growing threat to global health. The majority of TB cases occur in countries that lack diagnostic laboratory facilities. The requirement for biosafety level 3 facilities has hampered the availability of culture-confirmed diagnosis and drug susceptibility testing (DST) in many developing countries, with the result that only $5 \%$ of multidrug-resistant TB cases (MDR-TB) are identified and reported. ${ }^{1}$ To counter the lack of accessibility of DST, molecular methods are undergoing major developments. ${ }^{2}$

Rifampicin (RMP) is one of the main first-line antituberculosis drugs used in anti-tuberculosis treatment. Resistance to RMP is a potential marker of MDR-TB through mutation detection, mainly in the RMP resistance-determining region (RRDR) of the rpoB gene. ${ }^{3}$

The purpose of the present study was to evaluate the operational use of sodium hypochlorite $(\mathrm{NaOCl}$; household bleach) in sputum processing prior to DNA extraction and molecular analysis of RMP resistance. $\mathrm{NaOCl} 1 \%$ (1-10 min) has been shown to kill mycobacteria in sputum, ${ }^{4,5}$ and $\mathrm{NaOCl} 5 \%$ (5 min) to maintain DNA viability in body fluids. ${ }^{6}$ The study was carried out operationally at Cumura Hospital, Bissau, Guinea-Bissau; DST and molecular analysis were per- formed at the Fernando Fonseca Hospital, Amadora, and the National Health Institute, Lisbon, Portugal.

\section{MATERIALS AND METHODS}

At Cumura Hospital, TB is diagnosed essentially by clinical examination; the only routine laboratory method used is direct sputum smear microscopy. In this study, bleach processing was also performed on 74 sputum specimens (one per patient), as previously described, ${ }^{4}$ using $5 \% \mathrm{NaOCl}(15 \mathrm{~min})$ in screwcapped $15 \mathrm{ml}$ tubes, coupled with centrifugation to increase sensitivity. 4,7 The processed concentrate and the heat-fixed direct smears were stored at $-20^{\circ} \mathrm{C}$ until transportation at ambient temperature from $\mathrm{Cu}$ mura Hospital to Lisbon for Ziehl-Neelsen staining and grading of smears, ${ }^{8}$ both direct and from the processed concentrate, and for DNA extraction from the processed concentrate. Due to difficulties in transportation, DST was performed on only 20 unprocessed specimens sent to Lisbon. The study did not interfere with the diagnostic procedure routinely performed at Cumura Hospital. Full consent was provided by the hospital authorities.

DNA was obtained from the processed concentrate after heat lysis $\left(95^{\circ} \mathrm{C}, 15 \mathrm{~min}\right)$ using the

Correspondence to: S David, Instituto Nacional de Saúde Dr. Ricardo Jorge, Avenida Padre Cruz, 1649-016 Lisbon, Portugal. Tel: (+35) 1217519 200. Fax: (+35) 1217526 400. e-mail: david.suzana@gmail.com

Article submitted 26 September 2011. Final version accepted 9 May 2012. 